\begin{tabular}{|r|l|}
\hline \multicolumn{2}{|c|}{ Statistica Sinica Preprint No: SS-2020-0323 } \\
\hline Title & $\begin{array}{l}\text { On the Consistency of Least Squares Estimator in Models } \\
\text { Sampled at Random Times Driven by Long Memory } \\
\text { Noise: The Jittered Case }\end{array}$ \\
\hline Manuscript ID & SS-2020-0323 \\
\hline URL & http://www.stat.sinica.edu.tw/statistica/ \\
\hline DOI & $10.5705 /$ ss.202020.0323 \\
\hline Complete List of Authors & $\begin{array}{l}\text { Héctor Araya, } \\
\text { Natalia Bahamonde, } \\
\text { Lisandro Javier Fermín, } \\
\text { Tania Roa and } \\
\text { Soledad Torres }\end{array}$ \\
\hline Corresponding Author & Lisandro Javier Fermín \\
\hline E-mail & lisandro.fermin@uv.cl \\
\hline Notice: Accepted version subject to English editing.
\end{tabular}


Statistica Sinica

\title{
On the consistency of the least squares estimator in models sampled at random times driven by long memory noise: the Jittered case.
}

\author{
Héctor Araya ${ }^{\dagger}$, Natalia Bahamonde ${ }^{\ddagger}$, Lisandro Fermín*, Tania Roa* and Soledad Torres* \\ † Instituto de Estadística, Facultad de Ciencias, Universidad de Valparaíso \\ ¥ Instituto de Estadística, Pontificia Universidad Católica de Valparaíso \\ * CIMFAV - Instituto de Ingeniería Matemática, Universidad de Valparaíso \\ * Instituto de Matemáticas, Pontificia Universidad Católica de Valparaíso
}

\begin{abstract}
In numerous applications data are observed at random times. Our main purpose is to study a model observed at random times incorporating a long memory noise process with a fractional Brownian Hurst exponent $H$. In this article, we propose a least squares (LS) estimator in a linear regression model with long memory noise and a random sampling time called "jittered sampling". Specifically, there is a fixed sampling rate $1 / N$ but contaminated by an additive noise (the jitter) and governed by a probability density function supported in $[0,1 / N]$. The strong consistency of the estimator is established, with a convergence rate depending on $N$ and Hurst exponent. A Monte Carlo analysis supports the relevance of the theory and produces additional insights, with several levels of long-range dependence (varying the Hurst index) and two different jitter densities.
\end{abstract}

Key words and phrases: long memory noise, least squares estimator, random 
times, regression model.

\section{Introduction}

In different research areas, such as finance, network traffic, meteorology and astronomy among others, it has been noticed that the observations can be carried out sampling with random disturbances. Some examples of this sampling method are the data behavior until it is necessary to increase the sampling frequency, measurements obtained at random times, or defining stopping time when a particular event occurs, etc. In particular, in NietoBarajas and Sinha (2015) the authors discuss a Bayesian interpolation of unequally spaced time series. The case of paleoclimate time series was considered by Moerbeck et al. (2014) and Ólafsdóttir, KB., et al. (2016), where they estimate the significance of cross-correlations in unevenly sampled astronomical time series. Finally, in the area of computer science, we can mention the works given by Chang (2014) and Zhao, X., et al. (2014).

The study of statistical models in those situations is quite promising and has some open problems such as statistical inference and the limit behavior of the estimators.

In this article, we propose taking a first step in this direction; to study Least Squares (LS) estimator in a simple regression model with long mem- 
ory noise and observation measurements at random time.

Some previous works in this direction has been developed by Vilar (1995), the author studied the nonparametric kernel estimator of the regression function, $m(x)=\mathbb{E}(Y \mid X=x)$, under mixing dependence conditions, and the Ornstein-Uhlenbeck process driven by Brownian motion was studied by Vilar and Vilar (2000).

Also, Masry (1983) studied the problem of estimating an unknown probability density function on the based on $n$ independent observations sampled at random times.

Using a wavelet analysis Bardet and Bertrand (2010) studied the case of a nonparametric estimator of the spectral density of a Gaussian process with stationary increments, including the case of fractional Brownian motion, from the observation of one path at some particular class of random discrete times. They prove a central limit theorem providing an application to biological data.

Philippe, A., et al. (2020) give the latest works on this topic, the authors consider the study of the preservation of memory in a statistical model. With respect to the problem of parameter estimation in time series that may be represent as trend plus long memory noise, we can mention the works of Baillie and Chung (2002), Brockwell (2007), Lobato and Velasco 
(2002) among others.

We consider the Jittered sampling scheme that we define properly in Section 2. The term jitter is related to the temporal variability during the sending of digital signals or as the small variation in the accuracy of the clock signal, (we refer to Bellhouse (1981) and the references therein). It has also recently appeared in works related to the analysis of computational images, such as Khan (2017), Krune et al. (2016) and Subr et al. (2014)

Our main purpose, in studying a model with long memory noise, is the study of the characterization of the strong correlations between observations or persistence, by a slow decay of the correlations. To explain this phenomenon in a model, it is common to represent it through the Hurst exponent $H$, which takes values in $[0,1]$. In particular, the long-range dependence can be seen when $H \in(1 / 2,1)$. Since the work of Mandelbrot and Van Ness (1968) the effect of long-range dependence has been studied over years. One of the most popular Gaussian stochastic processes with long memory is the fractional Brownian motion. Some extensions to fBm with the same covariance structure are Rosenblatt, Tudor C.A. (2008), and Hermite, Tudor C.A. (2013), that we can consider here too.

With those motivations in mind, let us proceed to the mathematical description of the model we are dealing with. Namely, we consider the 
following simple regression model

$$
Y_{\tau_{i}}=a \tau_{i}+\Delta B_{\tau_{i}}^{H}, \quad i=0, \ldots, N-1
$$

where $a \in \mathbb{R}$ is the drift parameter of the model, $\Delta B_{\tau_{i}}^{H}=B_{\tau_{i+1}}^{H}-B_{\tau_{i}}^{H}$, and $\tau:=\left\{\tau_{i}, \quad 0 \leq i \leq N-1\right\}$ is the random time given by Jittered sampling.

The main interest in this work is to give a proof of the strong consistency for the least squares estimator in a random sampled linear regression model with long memory noise and an independent set of random times given by Jittered sampling. It is important to recall that the process $Y:=\left\{Y_{\tau_{i}}, 0 \leq\right.$ $i \leq N-1\}$ defined by equation (1.1) has long-range dependence and is non-stationary in the weak sense.

The plan of our paper is as follows. In Section 2 we present the definitions of the random times we are working within the random sampled regression model with long memory noise and we describe our notation. Section 3 is devoted to main results, we use least square procedure to get the parameter estimation and we analyse the almost sure convergence using Jittered sampling random time defined in the above section. In Section 4 , a simulation study is presented to illustrate the performance of the estimator, considering different values of $H$ and Jittered sampling random time. Finally in Section 5, we present the proof of Lemma 1, established in Section 3 and necessary to prove the almost sure convergence of the estimator. 


\section{Preliminaries}

In this section, we introduce the main tools from the stochastic calculus needed in the sequel. We present the random noise evaluated at the Jittered sampling random time that we considered throughout this work.

The long memory process, $B^{H}$, with Hurst parameter $H \in(1 / 2,1)$, is a centered process with the following properties:

(HN1) The covariance structure is given by:

$$
R_{H}(t, s):=\mathbb{E}\left[B_{t}^{H} B_{s}^{H}\right]=\frac{\sigma^{2}}{2}\left[t^{2 H}+s^{2 H}-|t-s|^{2 H}\right]
$$

(HN2) Is a self-similar process (with index $H$ ) with weakly stationary increments.

Remark 1. For example, $B^{H}$ can represent the well known fractional Brownian motion $(\mathrm{fBm})$. In $\mathrm{fBm}$ framework if $H=1 / 2$, then $B^{H}$ is a standard Brownian motion. Other types of long memory process with the same covariance structure as 2.1 are Hermite and Rosenblatt process. Also, the process $B^{H}$ is not a semimartingale if $H \neq 1 / 2$. Hence, we cannot apply the classical Itô calculus to $B^{H}$.

The random time sequence $\tau=\left\{\tau_{i} ; i=0, \ldots, N-1\right\}$ is strictly increasing; here $N$ represents the sample size and also the sampling frequency or 
sampling rate, that is the average number of samples obtained in $[0,1]$. In the sequel we will focus on the case where $\tau$ exhibits the following feature. Jittered sampling (JS): we assume that we observed a certain process at irregular times $\tau$, with period $\delta=1 / N>0$ but contaminated by an additive noise $\nu$, which represents possible measurement errors, satisfying the following hypothesis:

(HJ) $\nu=\left\{\nu_{i, N} ; \quad 0 \leq i \leq N-1\right\}$ is a sequence of independent and identically distributed random variables with common density function $g_{N}(\cdot)$, depending on $N$, with support on $[0,1 / N]$.

Thus, the sequence of random times $\tau_{i}, 0 \leq i \leq N-1$, is given by

$$
\tau_{i}=\frac{i}{N}+\nu_{i, N}, \quad i=0, \ldots, N-1
$$

Remark 2. Some examples of distributions that satisfy $(\mathbf{H J})$ are:

1. Uniform distribution on $[0,1 / N]$,

2. Triangular distribution with parameters $(0,1 / 2 N, 1 / N)$. The probability density function is given by

$$
f_{X}(x)= \begin{cases}0 & x<0 \\ 4 N^{2} x & 0 \leq x<\frac{1}{2 N} \\ 2 N & x=\frac{1}{2 N} \\ 4 N^{2}\left(\frac{1}{N}-x\right) & \frac{1}{2 N}<x \leq \frac{1}{N} .\end{cases}
$$


3. Raised Cosine distribution with parameters $\mu=1 / 2 N$ and $s=1 / 2 N$.

The probability density function is given by

$$
f_{X}(x)=N\left[1+\cos \left(\frac{x-1 / 2 N}{1 / 2 N} \pi\right)\right], \quad 0 \leq x \leq \frac{1}{N}
$$

Remark 3. It is important to notice that the hypothesis (HJ) implies $\mathbb{E}\left(\nu_{i, N}\right) \leq 1 / N$ and $\operatorname{Var}\left(\nu_{i, N}\right) \leq 1 / N^{2}$

Finally, we also assume the following hypothesis:

(HJN) The random time sequence $\tau$ and the long memory noise $B^{H}$ are independent.

Remark 4. In the deterministic case, i.e. $\tau_{i}=i / N, 0 \leq i \leq N$, for the Brownian motion case, $H=1 / 2$, the rate of the $L^{2}$-convergence for the least square estimator is obtained due to the property of independent increments. In fact, we have $\hat{a}_{N}-a=\frac{\sum_{i=0}^{N-1} \tau_{i} \Delta B_{\tau_{i}}}{\sum_{i=0}^{N-1} \tau_{i}^{2}}=\frac{6}{(N+1)(2 N+1)} \sum_{i=0}^{N-1} i \Delta B_{\tau_{i}}$ and

$$
\begin{aligned}
\mathbb{E}\left[\left(\hat{a}_{N}-a\right)^{2}\right] & =\mathbb{E}\left[\left(\frac{6}{(N+1)(2 N+1)} \sum_{i=0}^{N-1} i \Delta B_{\tau_{i}}\right)^{2}\right] \\
& =\frac{6}{(N+1)(2 N+1)} \leq \frac{6}{N^{2}}
\end{aligned}
$$

A direct application of Borel Cantelli lemma allow us to obtain the almost sure convergence of $\hat{a}_{N}$ to $a$. 
In the fractional Brownian motion case, increments are not longer independents. However for $H>1 / 2$, a simple modification in the procedure given in the main result (Theorem 1) of this article (Section 3) allow to obtain $L^{2}$ and almost sure convergence of $\hat{a}_{N}$ to $a$.

Particularly, in the work of Araya H.,et al. (2019), consistency of the drift estimated parameter is studied, when $\nu_{i, N}$ has an uniform distribution.

\section{Main results}

In this section, we provide our main results. We study the least squares estimation (LSE) for the random sampled linear regression model (1.1), with random times given by Jittered sampling and long memory noise. We prove that the LSE is an unbiased estimator for $a$ and that $\hat{a}_{N}$ converges, almost surely to $a$ (strongly consistent). It is worth mentioning that all the results in this article can be extended to a noise with the same covariance structure as the fractional Brownian motion, such as Rosenblatt and Hermite process.

For the estimation of the drift parameter $a$ in the model 1.1 , the least squares estimator is determined by

$$
\hat{a}_{N}=\frac{\sum_{i=0}^{N-1} \tau_{i} Y_{\tau_{i}}}{\sum_{i=0}^{N-1} \tau_{i}^{2}} .
$$


Recall that, from 1.1 and 3.1 we have,

$$
\hat{a}_{N}-a=\frac{\frac{1}{N} \sum_{i=0}^{N-1} \tau_{i} \Delta B_{\tau_{i}}^{H}}{\frac{1}{N} \sum_{i=0}^{N-1} \tau_{i}^{2}}:=\frac{A_{N}}{D_{N}} .
$$

To study the asymptotic behavior of (3.2), we will analyse separately the numerator and the denominator.

Remark 5. Since $1-\tau_{N-1} \leq 1 / N \rightarrow 0$ as $N \rightarrow \infty$. Then, almost sure convergence of $\tau_{N-1} \rightarrow 1$ is ensured as $N$ goes to infinity.

Theorem 1. Let $\tau$ be given by (2.2). Assume that the regression model (1.1) satisfies the hypothesis (HN1), (HN2), (HJ) and (HJN). Then, the LS estimator $\hat{a}_{N}$ given in (3.1) of the drift parameter a in the model (1.1) is strongly consistent, that is

$$
\hat{a}_{N} \underset{N \rightarrow \infty}{\stackrel{a . s .}{\longrightarrow}} a
$$

Proof. To prove our main theorem we need an auxiliary lemma related to the almost sure convergence of the denominator $D_{N}$ given in $(3.2)$. The proof of this lemma is indexed in Appendix 5 .

Lemma 1. Let $D_{N}$ be defined in (3.2). Let $\tau=\left\{\tau_{i} ; 0 \leq i \leq N-1\right\}$ the sampling random times defined by (2.2). If $\tau$ satisfies hypothesis (HJ), 
then

$$
D_{N} \underset{N \rightarrow \infty}{\stackrel{a . s .}{\longrightarrow}} \frac{1}{3}
$$

Remark 6. A direct computation gives the convergence of $D_{N} \rightarrow 1 / 3$ if we consider deterministic times $\tau_{i}=i / N$.

Hence, by Lemma 1, it remains to study the asymptotic behavior of $A_{N}$ as $N \rightarrow \infty$.

It is quite easy to see by the definition of $A_{N}$ and conditioning on $\tau$, that $\mathbb{E}\left[A_{N}\right]=0$.

Let us compute $\mathbb{E}\left[A_{N}^{2}\right]$.

$$
\begin{aligned}
\mathbb{E}\left[A_{N}^{2}\right] & =\mathbb{E}\left[\frac{1}{N^{2}} \sum_{i=0}^{N-1} \tau_{i}^{2}\left(B_{\tau_{i+1}}^{H}-B_{\tau_{i}}^{H}\right)^{2}\right] \\
& +\mathbb{E}\left[\frac{1}{N^{2}} \sum_{0 \leq i, j \leq N-1 ;|i-j|=1} \tau_{i} \tau_{j}\left(B_{\tau_{i+1}}^{H}-B_{\tau_{i}}^{H}\right)\left(B_{\tau_{j+1}}^{H}-B_{\tau_{j}}^{H}\right)\right] \\
& +\mathbb{E}\left[\frac{1}{N^{2}} \sum_{0 \leq i, j \leq N-1 ;|i-j| \geq 2} \tau_{i} \tau_{j}\left(B_{\tau_{i+1}}^{H}-B_{\tau_{i}}^{H}\right)\left(B_{\tau_{j+1}}^{H}-B_{\tau_{j}}^{H}\right)\right] \\
& :=\mathbb{E}\left[A_{N}^{(1)}\right]+\mathbb{E}\left[A_{N}^{(2)}\right]+\mathbb{E}\left[A_{N}^{(3)}\right],
\end{aligned}
$$

where we split the sum into three terms associated to the distance of the 
indexes. Firstly, we study the first term in 3.3 .

$$
\begin{aligned}
& \mathbb{E}\left[A_{N}^{(1)}\right] \\
& =\frac{1}{N^{2}} \sum_{i=0}^{N-1} \mathbb{E}\left[\mathbb{E}\left[\left|\frac{i}{N}+\nu_{i, N}\right|^{2}\left|B_{\frac{i+1}{N}+\nu_{i+1, N}}^{H}-B_{\frac{i}{N}+\nu_{i, N}}^{H}\right|^{2} \mid \nu_{i, N}=s_{i}, \nu_{i+1, N}=s_{i+1}\right]\right] \\
& =\frac{1}{N^{2}} \sum_{i=0}^{N-1} \int_{0}^{\frac{1}{N}} \int_{0}^{\frac{1}{N}}\left|\frac{i}{N}+s_{i}\right|^{2} \mathbb{E}\left[\left|B_{\frac{i+1}{N}+s_{i+1}}^{H}-B_{\frac{i}{N}+s_{i}}^{H}\right|^{2}\right] g_{N}\left(s_{i}\right) g_{N}\left(s_{i+1}\right) d s_{i} d s_{i+1} \\
& =\frac{1}{N^{2}} \sum_{i=0}^{N-1} \int_{0}^{\frac{1}{N}} \int_{0}^{\frac{1}{N}}\left|\frac{i}{N}+s_{i}\right|^{2}\left|\frac{i+1}{N}+s_{i+1}-\frac{i}{N}-s_{i}\right|^{2 H} g_{N}\left(s_{i}\right) g_{N}\left(s_{i+1}\right) d s_{i} d s_{i+1} \\
& =\frac{1}{N^{2}} \sum_{i=0}^{N-1} \int_{0}^{\frac{1}{N}} \int_{0}^{\frac{1}{N}}\left|\frac{i}{N}+s_{i}\right|^{2}\left|\frac{1}{N}+s_{i+1}-s_{i}\right|^{2 H} g_{N}\left(s_{i}\right) g_{N}\left(s_{i+1}\right) d s_{i} d s_{i+1} .
\end{aligned}
$$

From the properties of the long memory noise, due to hypothesis (HN1), (HN2) and (HJN), and since the domain of the random variables $\nu_{i, N}$ is $[0,1 / N]$, we obtain

$$
\begin{aligned}
\mathbb{E}\left[A_{N}^{(1)}\right] & \leq \frac{1}{N^{2}} \sum_{i=0}^{N-1} \int_{0}^{\frac{1}{N}} \int_{0}^{\frac{1}{N}}\left|\frac{i}{N}+\frac{1}{N}\right|^{2}\left|\frac{1}{N}+\frac{1}{N}\right|^{2 H} g_{N}\left(s_{i}\right) g_{N}\left(s_{i+1}\right) d s_{i} d s_{i+1} \\
& =\frac{1}{N^{2}} \sum_{i=0}^{N-1}\left|\frac{i}{N}+\frac{1}{N}\right|^{2}\left|\frac{1}{N}+\frac{1}{N}\right|^{2 H} \int_{0}^{\frac{1}{N}} \int_{0}^{\frac{1}{N}} g_{N}\left(s_{i}\right) g_{N}\left(s_{i+1}\right) d s_{i} d s_{i+1} \\
& \leq \frac{2^{2 H}}{N^{4+2 H}} \frac{N(N+1)(2 N+1)}{6} \leq \frac{C_{1}(H)}{N^{1+2 H}}
\end{aligned}
$$

with $C_{1}(H)=2^{2 H} / 3$.

Secondly, we consider the case of $|i-j|=1$ in $(3.3)$. For simplicity we take $j<i$, i.e. $j=i-1$, the other case can be treated in the same way. 
Therefore

$$
\begin{aligned}
& \mathbb{E}\left[A_{N}^{(2)}\right]=\frac{2}{N^{2}} \sum_{i=0}^{N-2} \mathbb{E}\left[\tau_{i+1} \tau_{i}\left(B_{\tau_{i+2}}^{H}-B_{\tau_{i+1}}^{H}\right)\left(B_{\tau_{i+1}}^{H}-B_{\tau_{i}}^{H}\right)\right] \\
= & \frac{2}{N^{2}} \sum_{i=0}^{N-2} \int_{0}^{\frac{1}{N}} \int_{0}^{\frac{1}{N}} \int_{0}^{\frac{1}{N}}\left(\frac{i+1}{N}+s_{i+1}\right)\left(\frac{i}{N}+s_{i}\right) \times \\
\mathbb{E} & {\left[\left(B_{\frac{i+2}{N}+s_{i+2}}^{H} B_{\frac{i+1}{N}+s_{i+1}}^{H}\right)\left(B_{\frac{i+1}{N}+s_{i+1}}^{H} B_{\frac{i}{N}+s_{i}}^{H}\right)\right] g_{N}\left(s_{i}\right) g_{N}\left(s_{i+1}\right) g_{N}\left(s_{i+2}\right) d s_{i} d s_{i+1} d s_{i+2}, }
\end{aligned}
$$

where in the last term we apply conditional expectation with respect to

$$
\begin{aligned}
& \nu_{i, N}=s_{i}, \nu_{i+1, N}=s_{i+1} \text { and } \nu_{i+2, N}=s_{i+2} \text {. Since } \\
& \mathbb{E}\left[\left(B_{\frac{i+2}{N}+s_{i+2}}^{H}-B_{\frac{i+1}{N}+s_{i+1}}^{H}\right)\left(B_{\frac{i+1}{N}+s_{i+1}}^{H}-B_{\frac{i}{N}+s_{i}}^{H}\right)\right] \\
& \quad=\frac{1}{2}\left[\left|s_{i+2}-s_{i}+\frac{2}{N}\right|^{2 H}-\left|s_{i+2}-s_{i+1}+\frac{1}{N}\right|^{2 H}-\left|s_{i+1}-s_{i}+\frac{1}{N}\right|^{2 H}\right] .
\end{aligned}
$$

Then

$$
\mathbb{E}\left[\left(B_{\frac{i+2}{N}+s_{i+2}}^{H}-B_{\frac{i+1}{N}+s_{i+1}}^{H}\right)\left(B_{\frac{i+1}{N}+s_{i+1}}^{H}-B_{\frac{i}{N}+s_{i}}^{H}\right)\right] \leq \frac{3^{2 H}}{2 N^{2 H}},
$$

Plugging inequality (3.6) into the equation 3.5 yields

$$
\begin{aligned}
& \mathbb{E}\left[A_{N}^{(2)}\right] \leq \frac{2}{N^{2}} \sum_{i=0}^{N-2} \int_{0}^{\frac{1}{N}} \int_{0}^{\frac{1}{N}} \int_{0}^{\frac{1}{N}}\left[\frac{i+1}{N}+s_{i+1}\right]\left[\frac{i}{N}+s_{i}\right] \frac{3^{2 H}}{2 N^{2 H}} \\
& \times g_{N}\left(s_{i}\right) g_{N}\left(s_{i+1}\right) g_{N}\left(s_{i+2}\right) d s_{i} d s_{i+1} d s_{i+2} \\
& \leq \frac{3^{2 H}}{N^{2+2 H}} \sum_{i=0}^{N-2} \int_{0}^{\frac{1}{N}} \int_{0}^{\frac{1}{N}} \int_{0}^{\frac{1}{N}}\left[\frac{i+1}{N}+\frac{1}{N}\right]\left[\frac{i}{N}+\frac{1}{N}\right] \\
& \times g_{N}\left(s_{i}\right) g_{N}\left(s_{i+1}\right) g_{N}\left(s_{i+2}\right) d s_{i} d s_{i+1} d s_{i+2} \\
& =\frac{3^{2 H}}{N^{4+2 H}} \sum_{i=0}^{N-2}(i+2)(i+1) \leq \frac{3^{2 H}}{3 N^{4+2 H}}(N-1) N(N+1) \\
& \leq \frac{C_{2}(H)}{N^{1+2 H}}
\end{aligned}
$$


being $C_{2}(H)=3^{2 H-1}$. Finally we consider the case $|i-j| \geq 2$ in $(3.3)$.

Conditioning on $\nu_{i, N}=s_{i}, \nu_{i+1 ; N}=s_{i+1}, \nu_{j, N}=s_{j}$ and $\nu_{j+1, N}=s_{j+1}$, we get

$$
\begin{aligned}
& \mathbb{E}\left[A_{N}^{(3)}\right]=\frac{1}{N^{2}} \mathbb{E}\left[\sum_{0 \leq i, j \leq N-1 ;|i-j| \geq 2} \tau_{i} \tau_{j}\left(B_{\tau_{i+1}}^{H}-B_{\tau_{i}}^{H}\right)\left(B_{\tau_{j+1}}^{H}-B_{\tau_{j}}^{H}\right)\right] \\
& =\frac{1}{N^{2}} \sum_{0 \leq i, j \leq N-1 ;|i-j| \geq 2} \int_{0}^{\frac{1}{N}} \int_{0}^{\frac{1}{N}} \int_{0}^{\frac{1}{N}} \int_{0}^{\frac{1}{N}}\left(\frac{i}{N}+s_{i}\right)\left(\frac{j}{N}+s_{j}\right) \\
& \times \mathbb{E}\left[\left(B_{\frac{i+1}{N}+s_{i+1}}^{H}-B_{\frac{i}{N}+s_{i}}^{H}\right)\left(B_{\frac{j+1}{N}+s_{j+1}}^{H}-B_{\frac{j}{N}+s_{j}}^{H}\right)\right] \\
& \times g_{N}\left(s_{i}\right) g_{N}\left(s_{i+1}\right) g_{N}\left(s_{j}\right) g_{N}\left(s_{j+1}\right) d s_{i} d s_{i+1} d s_{j} d s_{j+1} \\
& =\frac{1}{N^{2}} \sum_{0 \leq i, j \leq N-1 ;|i-j| \geq 2} \int_{0}^{\frac{1}{N}} \int_{0}^{\frac{1}{N}} \int_{0}^{\frac{1}{N}} \int_{0}^{\frac{1}{N}}\left(\frac{i}{N}+s_{i}\right)\left(\frac{j}{N}+s_{j}\right) \\
& \times \mathbf{I}_{\mathbf{i}, \mathbf{j}} g_{N}\left(s_{i}\right) g_{N}\left(s_{i+1}\right) g_{N}\left(s_{j}\right) g_{N}\left(s_{j+1}\right) d s_{i} d s_{i+1} d s_{j} d s_{j+1}
\end{aligned}
$$

where

$$
\begin{aligned}
& \mathbf{I}_{\mathbf{i}, \mathbf{j}}:=\mathbb{E}\left[\left(B_{\frac{i+1}{N}+s_{i+1}}^{H}-B_{\left.\frac{i}{N}+s_{i}\right)}^{H}\right)\left(B_{\frac{j+1}{N}+s_{j+1}}^{H}-B_{\frac{j}{N}+s_{j}}^{H}\right)\right] \\
& =\frac{1}{2}\left[\left|\frac{i-j+1}{N}+s_{i+1}-s_{j}\right|^{2 H}+\left|\frac{i-j-1}{N}+s_{i}-s_{j+1}\right|^{2 H}\right. \\
& \left.-\left|\frac{i-j}{N}+s_{i+1}-s_{j+1}\right|^{2 H}-\left|\frac{i-j}{N}+s_{i}-s_{j}\right|^{2 H}\right] .
\end{aligned}
$$


For $i-j=2$ (equivalently for $i-j=-2$ ) we directly get:

$$
\begin{aligned}
& \mathbf{I}_{\mathbf{i}, \mathbf{j}}:=\frac{1}{2}\left[\left|\frac{3}{N}+s_{i+1}-s_{j}\right|^{2 H}+\left|\frac{1}{N}+s_{i}-s_{j+1}\right|^{2 H}\right. \\
& \left.-\left|\frac{2}{N}+s_{i+1}-s_{j+1}\right|^{2 H}-\left|\frac{}{N}+s_{i}-s_{j}\right|^{2 H}\right] \\
& \leq \frac{1}{2}\left[\left|\frac{3}{N}+s_{i+1}-s_{j}\right|^{2 H}+\left|\frac{1}{N}+s_{i}-s_{j+1}\right|^{2 H}\right] \\
& \leq \frac{1}{2}\left[\left|\frac{4}{N}\right|^{2 H}+\left|\frac{2}{N}\right|^{2 H}\right]=\frac{2^{2 H-1}\left(2^{2 H}+1\right)}{N^{2 H}} .
\end{aligned}
$$

For $|i-j|>2$, applying Taylor expansion to the function $x^{2 H}$ allows to get

$$
\begin{aligned}
& \left|\frac{i-j+1}{N}+s_{i+1}-s_{j}\right|^{2 H}-\left|\frac{i-j}{N}+s_{i}-s_{j}\right|^{2 H} \\
& =2 H\left|\frac{i-j}{N}+s_{i}-s_{j}\right|^{2 H-1}\left(s_{i+1}-s_{i}+\frac{1}{N}\right)+R_{N}^{1}
\end{aligned}
$$

and

$$
\begin{aligned}
& \left|\frac{i-j}{N}+s_{i+1}-s_{j+1}\right|^{2 H}-\left|\frac{i-j-1}{N}+s_{i}-s_{j+1}\right|^{2 H} \\
& =2 H\left|\frac{i-j-1}{N}+s_{i}-s_{j+1}\right|^{2 H-1}\left(s_{i+1}-s_{i}+\frac{1}{N}\right)+R_{N}^{2} .
\end{aligned}
$$

Therefore

$$
\begin{aligned}
\mathbf{I}_{\mathbf{i}, \mathbf{j}} & =2 H\left(s_{i+1}-s_{i}+\frac{1}{N}\right)\left[\left|\frac{i-j}{N}+s_{i}-s_{j}\right|^{2 H-1}\right. \\
& \left.-\left|\frac{i-j-1}{N}+s_{i}-s_{j+1}\right|^{2 H-1}\right]+R_{N}^{1}-R_{N}^{2} .
\end{aligned}
$$


Again, applying Taylor theorem to the function $x^{2 H-1}$, we obtain

$$
\begin{aligned}
\mathbf{I}_{\mathbf{i}, \mathbf{j}} & =2 H(2 H-1)\left(s_{i+1}-s_{i}+\frac{1}{N}\right)\left(s_{j+1}-s_{j}+\frac{1}{N}\right) \\
& \times\left|\frac{i-j-1}{N}+s_{i}-s_{j+1}\right|^{2 H-2}+R_{N}^{3}+R_{N}^{1}-R_{N}^{2},
\end{aligned}
$$

which implies by the fact that $2 H-2<0$, for $|i-j|>2$

$$
\mathbf{I}_{\mathbf{i}, \mathbf{j}} \leq \frac{C_{3}(H)}{N^{2}}\left|\frac{i-j-1}{N}+s_{i}-s_{j+1}\right|^{2 H-2} \leq \frac{C_{3}(H)}{N^{2 H}}
$$

where $C_{3}(H)=8 H(2 H-1)$. Notice here that the remainder terms $R_{N}^{1}, R_{N}^{2}$ and $R_{N}^{3}$ are of order $N^{-2 H}$ and uniformly independent on $i$ and $j$. Plugging (3.9) and 3.10 into the expression 3.8 and considering $C_{4}(H)=$ $C_{3}(H)+2^{2 H-1}\left(2^{2 H}+1\right)$ we obtain

$$
\begin{aligned}
\mathbb{E}\left[A_{N}^{(3)}\right] \leq \frac{C_{4}(H)}{N^{2 H+2}} \sum_{0 \leq i, j \leq N-1 ;|i-j| \geq 2} \int_{0}^{1 / N} \int_{0}^{1 / N} \int_{0}^{1 / N} \int_{0}^{1 / N}\left(\frac{i}{N}+s_{i}\right)\left(\frac{j}{N}+s_{j}\right) \\
\quad \times g_{N}\left(s_{i}\right) g_{N}\left(s_{i+1}\right) g_{N}\left(s_{j}\right) g_{N}\left(s_{j+1}\right) d s_{i} d s_{i+1} d s_{j} d s_{j+1} \\
\quad \leq \frac{C_{4}(H)}{N^{2 H+2}} \sum_{0 \leq i, j \leq N-1 ;|i-j| \geq 2}\left(\frac{i+1}{N}\right)\left(\frac{j+1}{N}\right) .
\end{aligned}
$$

Moreover, the above sum in (3.11) can be computed as follows

$$
\frac{1}{N^{2}} \sum_{0 \leq i, j \leq N-1 ;|i-j| \geq 2}\left(\frac{i+1}{N}\right)\left(\frac{j+1}{N}\right)=\frac{1}{N^{4}}\left(\frac{N^{4}}{4}-\frac{N^{3}}{6}-\frac{N^{2}}{4}+\frac{N}{6}\right) \leq \frac{1}{4}
$$

Therefore

$$
\mathbb{E}\left[A_{N}^{(3)}\right] \leq \frac{C_{5}(H)}{N^{2 H}}
$$


where $C_{5}(H)=C_{4}(H) / 4$. Substituting (3.4), (3.7) and 3.12 into the equation in 3.3 we obtain

$$
\mathbb{E}\left[\left(A_{N}\right)^{2}\right] \leq \frac{C_{1}(H)+C_{2}(H)}{N^{1+2 H}}+\frac{C_{5}(H)}{N^{2 H}}
$$

Since $H>1 / 2$, then the $L^{2}$ rate of $A_{N}$ is faster than $1 / N$. A direct application of Borell-Cantelli lemma allow us to obtain $A_{N} \underset{N \rightarrow \infty}{\stackrel{a . s .}{\longrightarrow}} 0$.

\section{Remark 7.}

Under random sampling scheme, the $L^{2}$-convergence is of order $1 / N^{2 H}$. Borel Cantelli lemma allows us to get the a.s. convergence of order $1 / N^{2 H-1}$.

When $\tau_{i}=i / N$, from remark 6 and with a small modification, we can ensure the same $L^{2}$ and a.s. convergence given in Theorem 1 .

For $H<1 / 2$ (anti persistent case), the same arguments as before assure us that $A_{N}$ converges to zero in $L^{2}$, and then in probability. Also $D_{N}$ converges almost surely to $1 / 3$, and therefore in probability. Using Slutsky's Theorem we obtain the convergence in probability of $\hat{a}_{N}$ to $a$. However our method not allowed to obtain the almost sure convergence in this case $(H<1 / 2)$. 


\section{Simulation Study}

In this section, we present Monte Carlo simulation study to evaluate the performance of the least squares estimator for a finite sample $(N=10,15,20,25$, 50,75 and 100) in linear regression model (1.1). We also perform a study for a large sample size $(\mathrm{N}=300)$. In both cases 1000 replicates of the $\hat{a}_{N}$ are studied.

The deterministic case: We consider the model defined by equation (1.1) observed at equally spaced times, i.e. $\tau_{i}=i / N, i=0, \ldots, N-1$.

The uniform and triangular case: We review parametric estimation when the model is observed at sampling random times and considering the uniform and triangular distributions, both with support on $[0,1 / N]$.

\subsection{Small sample size}

When considering the jittered sampling scheme, the squared error decreases as the sample size increases. Figure 1 and 2 show boxplots of $M=1000$ trials of $\hat{a}_{N}$ for $N=10,15,20,25,50,75,100$, for three different values of $H$, under three different scenarios (deterministic, uniform and triangular case) and for initial values of $a=0.2$ and $a=2$. 

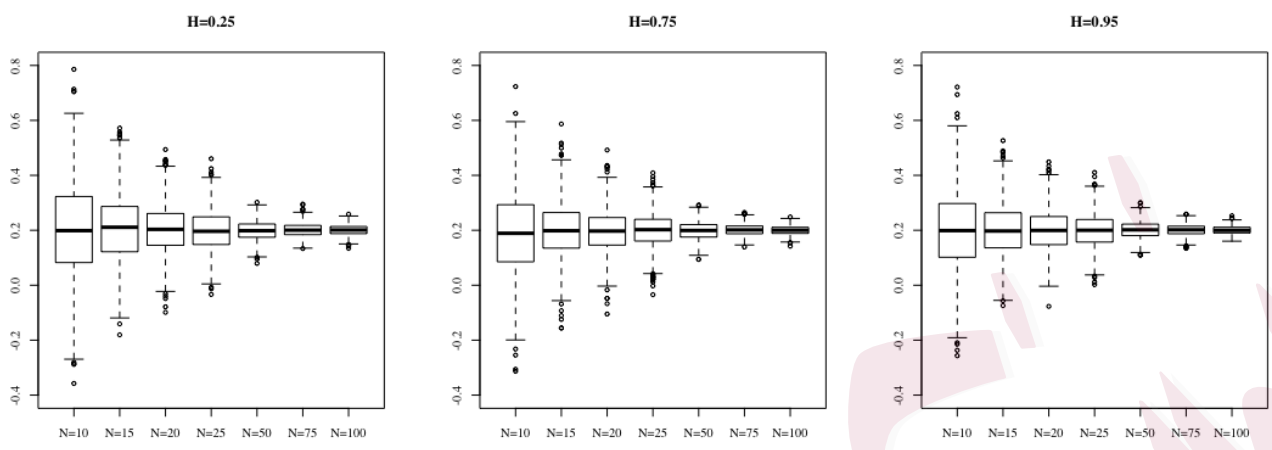

(a) Deterministic
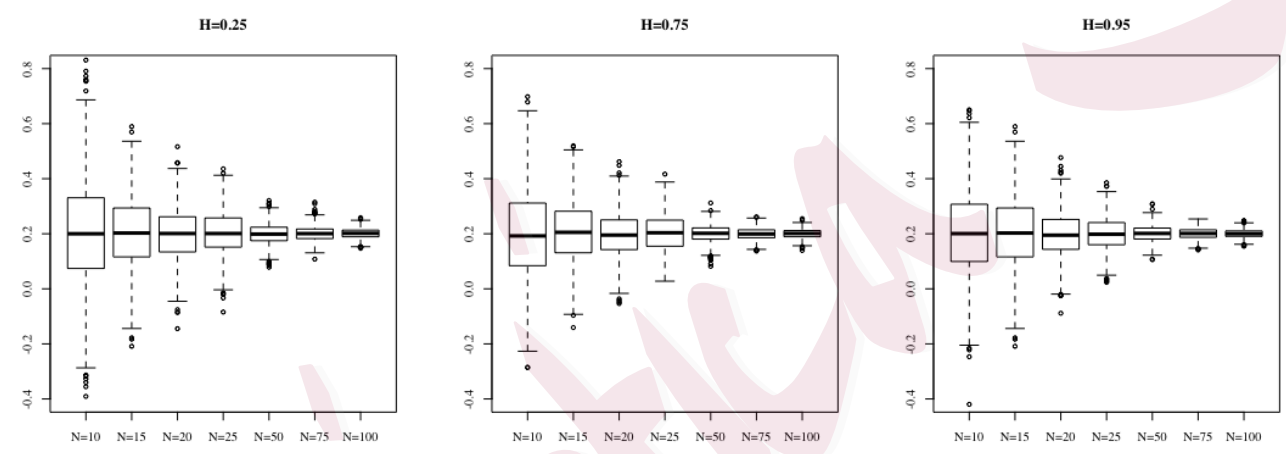

(b) Uniform 

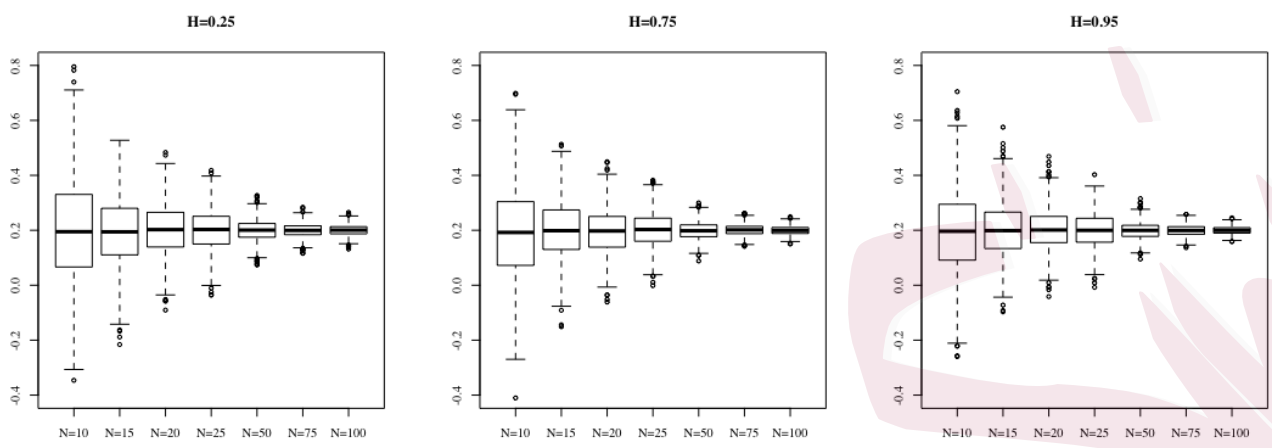

(c) Triangular

Figure 1: Boxplots for $\hat{a}_{N}$ when $N=10,15,20,25,50,75,100$, different values of $H$, under deterministic, uniform and deterministic case for $a=0.2$
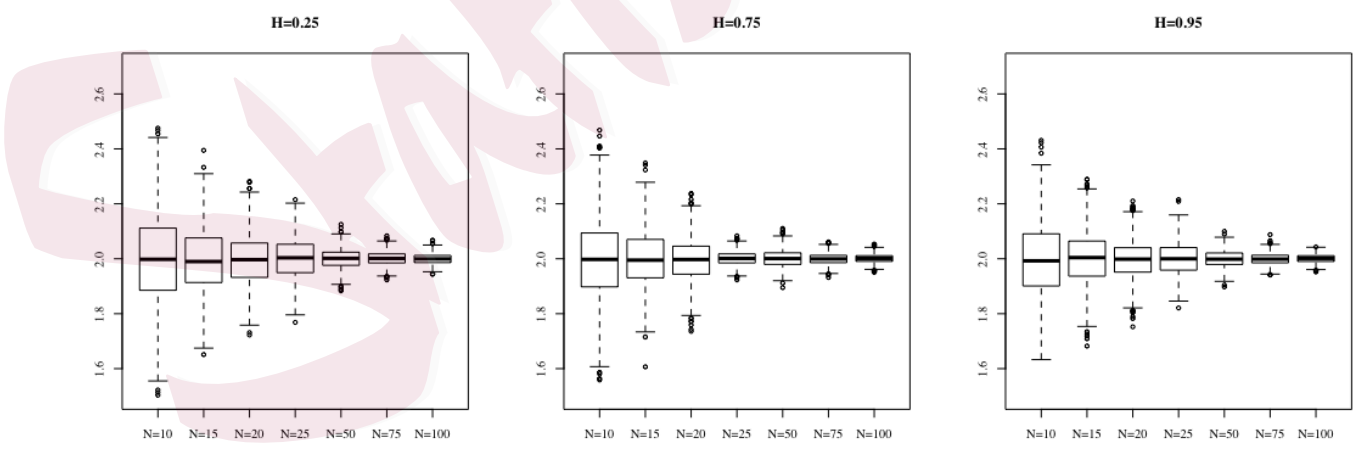

(a) Deterministic 


\subsection{Small sample size21}

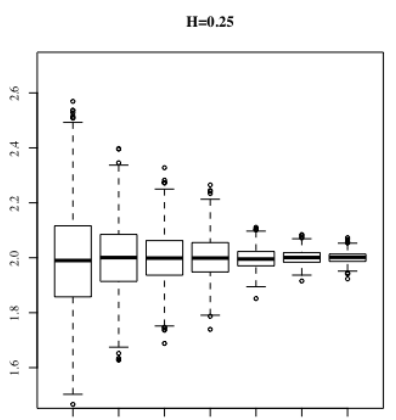

$N=10 \quad N=15 \quad N=20 \quad N=25 \quad N=50 \quad N=75 \quad N=100$

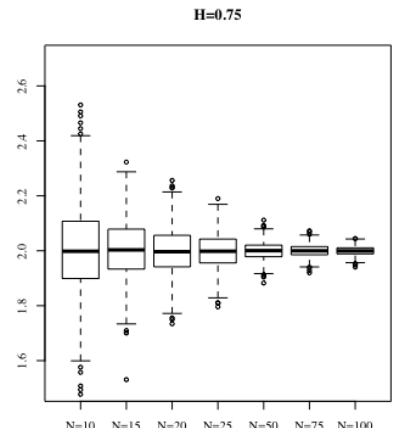

$\begin{array}{llllllll}N=10 & N=15 & N=20 & N=25 & N=50 & N=75 & N=100\end{array}$

(b) Uniform
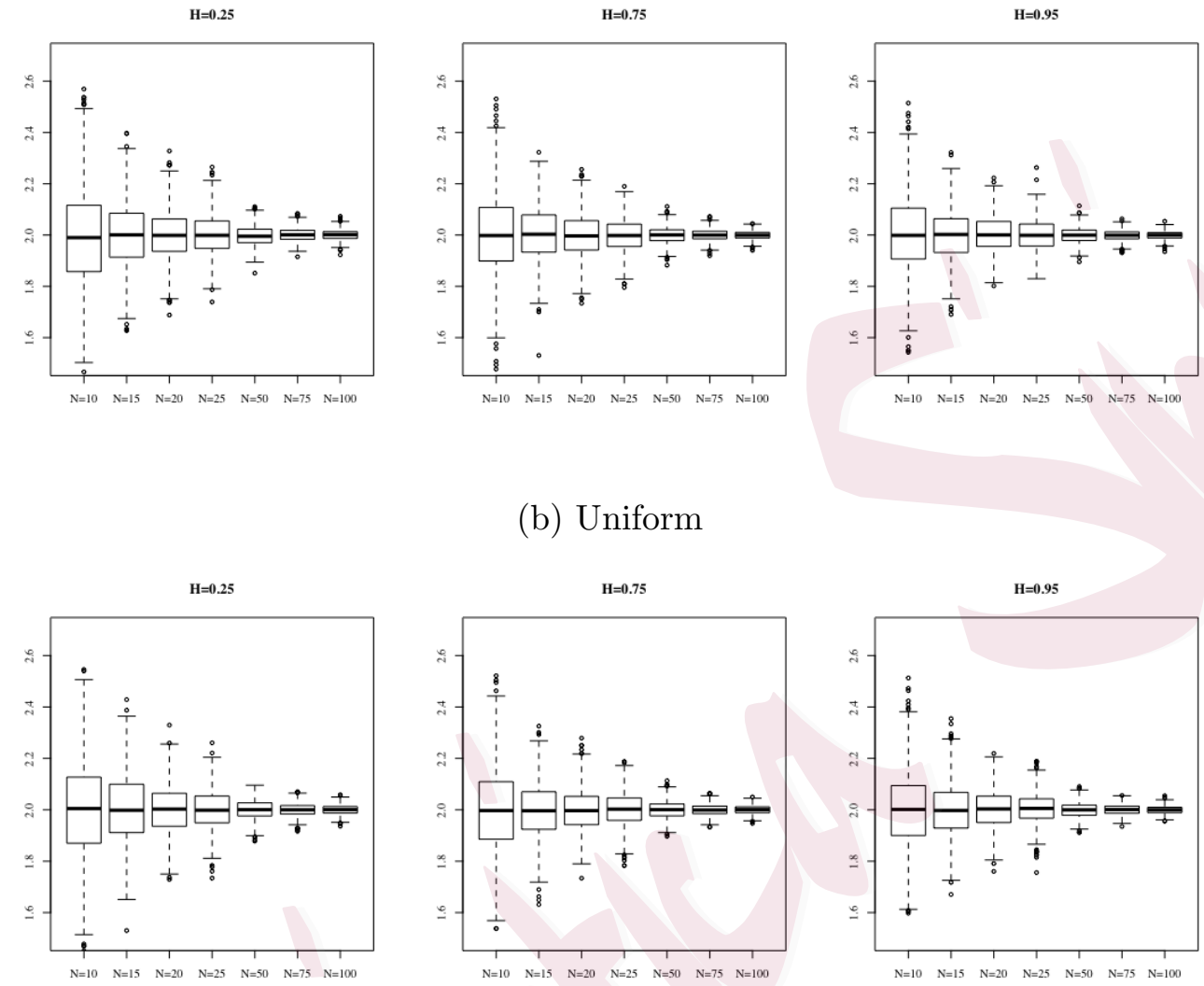

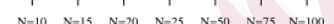

(c) Triangular

Figure 2: Boxplots for $\hat{a}_{N}$ when $N=10,15,20,25,50,75,100$, different values of $H$, under deterministic, uniform and deterministic case for $a=2$ 
As $N$ increases the variability of $\hat{a}_{N}$, around the real value of the parameter, decreases; this situation holds in the same way for different values of $H$ and for the different cases considered. There is a remarkable reduction of the interquartile range for the three different scenarios considered as the value of $N$ increases. From $(3.13)$ we have that the upper bound of the convergence rate of $\left|\hat{a}_{N}-a\right|$ is $C / N^{2 H-1}$, for almost sure convergence, and $1 / N^{2 H}$ for convergence in probability. This upper bound is obtained for each fix $N$. We can note that as long as $H$ and $N$ values increases the estimation of the real parameter is more accurate, even for small values of $N$ the estimation is close to the real parameter.

\subsection{Large sample size}

For all the simulations shown, we consider $M=1000$ replicates of the model with the parameter $a=0.2$ and $a=2$, different values of $N$ from $N=3$ to $N=300$. We also consider different values of the Hurst parameter: $H=0.05, H=0.25$ and $H=0.45$ (anti persistent cases); $H=0.55$, $H=0.75$ and 0.95 (long memory cases). For a practical reason, in Figure 3 four different values of $H$ have been considered for the graphs, in Figure 4 three different values of $H$ have been considered. While, for the tables, all the aforementioned values of $H$ have been considered. 


\subsection{Large sample size23}

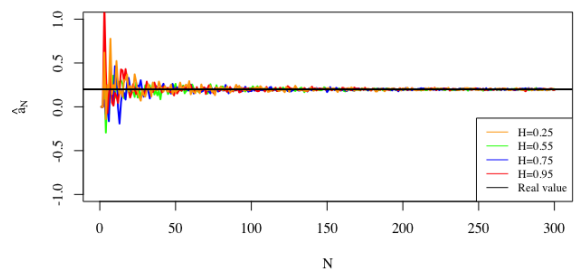

(a) Uniform, $a=0.2$.

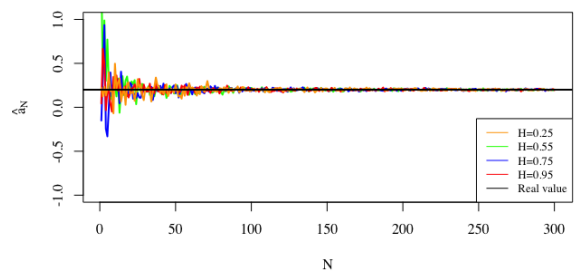

(c) Triangular, $a=0.2$.

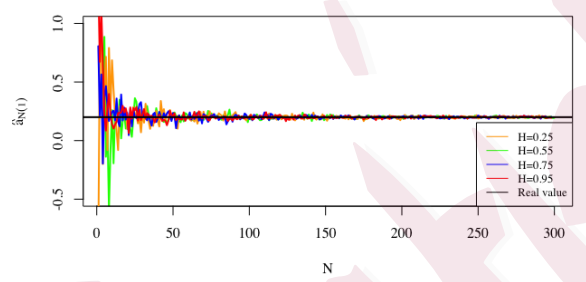

(e) Deterministic, $a=0.2$.

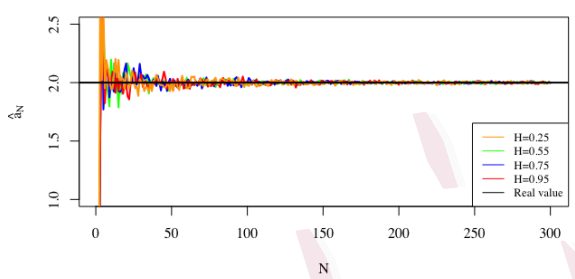

(b) Uniform, $a=2$.

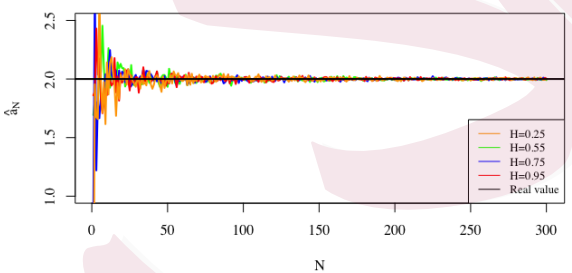

(d) Triangular, $a=2$.

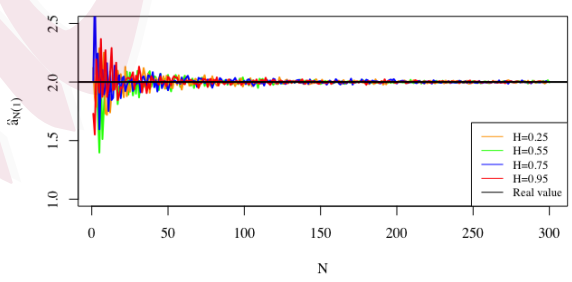

(f) Deterministic, $a=2$.

Figure 3: Rate of convergence of $\hat{a}_{N}$ under deterministic, uniform and triangular distribution and different values of $H$ : (left) case $a=0.2$, (right) case $a=2$. 


\subsection{Large sample size24}

Figure 3 shows the value of $\hat{a}_{N}$ for each value of $N$ from 3 to 300 , for all different values of $H$, the true value of the parameter is reached, even when the noise, driven by an anti persistent process $(H=0.25)$, is considered. We can see that for the case of $a=0.2$ and $a=2$, the uniform scheme converges faster to the real parameter. This confirms the discussion on convergence speed of the different values of $H$ and shows that the explicit scheme can perform better for some selections of the long memory parameter. Table 1 and 2 summarize the simulation results for estimation according to equation (3.1). Performance statistics presented are the mean, standard deviation (SD) and kurtosis from $M=1000$ trials for $N=300$ fixed, of $\hat{a}_{N}$. The kurtosis refers to the difference between kurtosis of a Gaussian distribution and that of the random variable $a_{N}$. 
4.2 Large sample size25

\begin{tabular}{ccccccc}
\hline Deterministic & $H=0.05$ & $H=0.25$ & $H=0.45$ & $H=0.55$ & $H=0.75$ & $H=0.95$ \\
\hline Mean & 0.1998 & 0.1997 & 0.2000 & 0.2001 & 0.1997 & 0.2001 \\
SD & 0.0070 & 0.0061 & 0.0059 & 0.0056 & 0.0053 & 0.0049 \\
Kurtosis & -0.0653 & 0.0631 & -0.1805 & -0.1815 & 0.0401 & -0.0528 \\
\hline Uniform & $H=0.05$ & $H=0.25$ & $H=0.45$ & $H=0.55$ & $H=0.75$ & $H=0.95$ \\
\hline Mean & 0.1996 & 0.2000 & 0.1998 & 0.2003 & 0.2001 & 0.1999 \\
SD & 0.0069 & 0.0061 & 0.0057 & 0.0054 & 0.0053 & 0.0050 \\
Kurtosis & 0.0058 & -0.0309 & -0.2094 & -0.2633 & -0.0163 & -0.2246 \\
\hline Triangular & $H=0.05$ & $H=0.25$ & $H=0.45$ & $H=0.55$ & $H=0.75$ & $H=0.95$ \\
\hline Mean & 2.0002 & 1.9996 & 2.0005 & 1.9997 & 2.0002 & 2.0001 \\
SD & 0.0070 & 0.0060 & 0.0058 & 0.0059 & 0.0052 & 0.0051 \\
Kurtosis & -0.0400 & -0.1758 & 0.0772 & 0.3426 & -0.0475 & -0.0785 \\
\hline
\end{tabular}

Table 1: Uniform and triangular case: Mean, SD and kurtosis with $a=0.2$.

Overall, for all $H$ 's values it is possible to notice that SD decreases as the value of $H$ increases, which is expected since, in the context of longrange dependence processes, it is quite common for the process to be less noisy. This is a reflection of the consistency of the estimator. On the other hand, when $H$ increasing towards 1 , the empirical estimation has a better behavior which is reflected in a more accurate solution. 
4.2 Large sample size26

\begin{tabular}{ccccccc}
\hline Deterministic & $H=0.05$ & $H=0.25$ & $H=0.45$ & $H=0.55$ & $H=0.75$ & $H=0.95$ \\
\hline Mean & 2.000 & 2.000 & 2.000 & 1.9998 & 2.0000 & 1.9998 \\
SD & 0.0069 & 0.0062 & 0.0060 & 0.0057 & 0.0054 & 0.0050 \\
Kurtosis & -0.0804 & -0.0948 & -0.0326 & 0.1986 & 0.0797 & -0.0822 \\
\hline Uniform & $H=0.05$ & $H=0.25$ & $H=0.45$ & $H=0.55$ & $H=0.75$ & $H=0.95$ \\
\hline Mean & 1.9999 & 2.0004 & 2.0003 & 2.0001 & 2.0002 & 2.0001 \\
SD & 0.0069 & 0.0061 & 0.0060 & 0.0056 & 0.0054 & 0.0049 \\
Kurtosis & 0.0401 & -0.1856 & -0.2662 & 0.0499 & -0.0646 & 0.0181 \\
\hline Triangular & $H=0.05$ & $H=0.25$ & $H=0.45$ & $H=0.55$ & $H=0.75$ & $H=0.95$ \\
\hline Mean & 2.0002 & 1.9996 & 2.0005 & 1.9997 & 2.0002 & 2.0001 \\
SD & 0.0070 & 0.0060 & 0.0058 & 0.0059 & 0.0052 & 0.0051 \\
Kurtosis & -0.0400 & -0.1758 & 0.0772 & 0.3426 & -0.0475 & -0.0785 \\
\hline
\end{tabular}

Table 2: Uniform and triangular case: Mean, SD and kurtosis with $a=2$.

Figure 4 show the frequency histograms (sampling distribution) of $M=$ 1000 values of the variable $N\left(\hat{a}_{N}-a\right)$ generated for different values of $H$. We take the values $a=0.2$ and $a=2$ and the random times follow uniform and triangular distributions, respectively.

The empirical (red line) and theoretical (green line) distributions are overlapped. The theoretical distribution considered is a normal distribution 


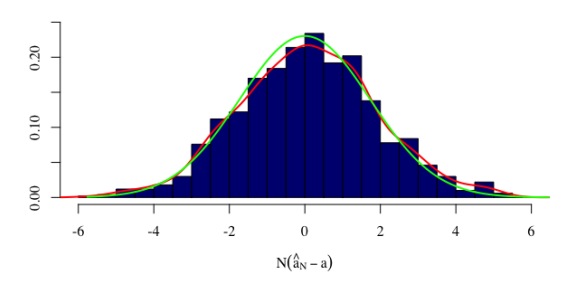

(a) $H=0.25$

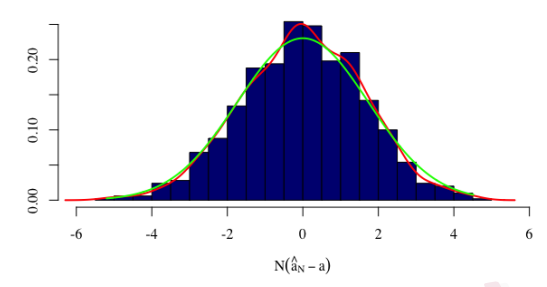

(c) $H=0.75$

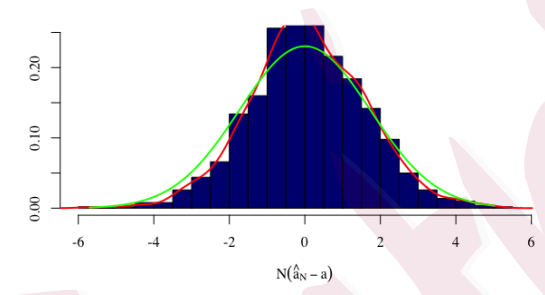

(e) $H=0.95$

Uniform distribution

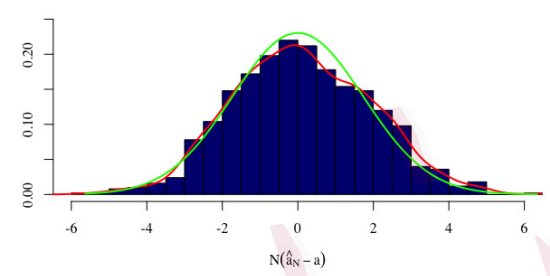

(b) $H=0.25$

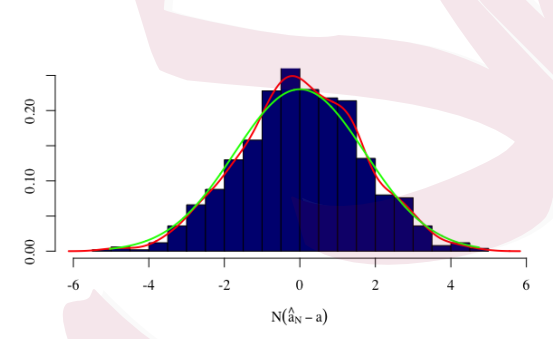

(d) $H=0.75$

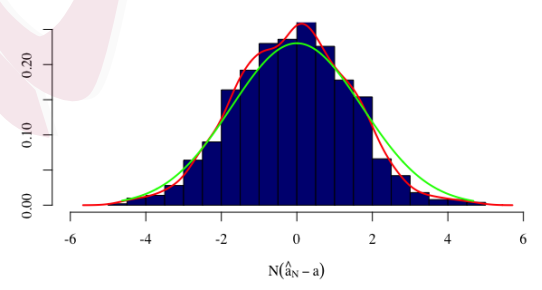

(f) $H=0.95$

Triangular distribution

Figure 4: (Left) Histograms for $N\left(\hat{a}_{N}-0.2\right)$ under uniform distribution and different values of $H$. (Right) Histograms for $N\left(\hat{a}_{N}-2\right)$ under triangular distribution and different values of $H$. 
with parameters $\mu=0$ and $\sigma^{2}=3$. The value of $\sigma^{2}$ comes from Roa, T., et al. (2020), where the authors consider the case $H=1 / 2$, the Brownian motion, and they prove that in this case $N\left(\hat{a}_{N}-a\right)$ converges in distribution to a $N(0,3)$. We emphasize here that computing the asymptotic distributions in a general case is a challenge in itself and could represent another task. In conclusion, we showed that the empirical estimation of the least squares estimator in models driven by long memory noise, under random scheme described by (2.2) guarantees stability and convergence, is very accurate for any value $H \in(0,1)$ and different values of $a$. Furthermore, when $H$ increases towards 1 the estimator presents less variability, the same conclusion can be made when $N$ approaches to infinity. Therefore, the estimation procedure studied is a good alternative to estimate parameters in a linear regression model with random times and long range dependent noise.

\section{Appendix: Proof of Lemma 1}

Proof. By definition of $D_{N}$ given in equation (3.2), we have

$$
\begin{aligned}
D_{N} & =\frac{1}{N} \sum_{i=0}^{N-1}\left(\frac{i}{N}+\nu_{i, N}\right)^{2} \\
& =\frac{1}{N} \sum_{i=0}^{N-1} \frac{i^{2}}{N^{2}}+\frac{2}{N} \sum_{i=0}^{N-1} \frac{i \nu_{i, N}}{N}+\frac{1}{N} \sum_{i=0}^{N-1} \nu_{i, N}^{2} \\
& =I_{N}^{(1)}+I_{N}^{(2)}+I_{N}^{(3)}
\end{aligned}
$$


First, $I_{N}^{(1)}=\frac{1}{N^{3}} \sum_{i=0}^{N-1} i^{2}=\frac{2 N^{3}-3 N^{2}+N}{6 N^{3}}$. Then,

$$
\lim _{N \rightarrow \infty} I_{N}^{(1)}=\frac{1}{3}
$$

Now for $I_{N}^{(2)}$, let $\gamma>0$ we use the fact that $0 \leq \nu_{i, N} \leq 1 / N$ for all $i=0, \ldots, N-1$. Now, we will apply Chebyshev inequality as follows:

$$
\begin{aligned}
\mathbb{P}\left(\left|\frac{2}{N^{2}} \sum_{i=0}^{N-1} i \nu_{i, N}\right|>\frac{1}{N^{\gamma}}\right) & \leq \frac{1}{N^{-2 \gamma}} \mathbb{E}\left[\left(\frac{2}{N^{2}} \sum_{i=0}^{N-1} i \nu_{i, N}\right)^{2}\right] \\
& =\frac{4}{N^{4-2 \gamma}} \mathbb{E}\left[\left(\sum_{i=0}^{N-1} i \nu_{i, N}\right)^{2}\right] \\
& \leq \frac{4}{N^{4-2 \gamma}}\left(\sum_{i=0}^{N-1} \frac{i}{N}\right)^{2} \\
& =\frac{4}{N^{4-2 \gamma}}\left(\frac{N-1}{2}\right)^{2} \\
& \leq \frac{1}{N^{2-2 \gamma}}
\end{aligned}
$$

Given that $\nu_{i, N}$ is a sequence of events in a probability space we are in position to use Borel Cantelli Lemma; we need to find a strictly positive $\gamma$, so that

$$
\sum_{N \geq 1} \mathbb{P}\left(\left|\frac{2}{N^{2}} \sum_{i=0}^{N-1} i \nu_{i, N}\right|>\frac{1}{N^{\gamma}}\right) \leq \sum_{N \geq 1} \frac{1}{N^{2-2 \gamma}}<\infty
$$

to ensure the convergence of the previous sum, it is necessary to find a value 
for $\gamma$ such that $2-2 \gamma>1$, for $0<\gamma<1 / 2$, then

$$
I_{N}^{(2)}=\frac{2}{N} \sum_{i=0}^{N-1} \frac{i \nu_{i, N}}{N} \underset{N \rightarrow \infty}{a . s .} 0 .
$$

For the third term $I_{N}^{(3)}$, we take into account that $\nu_{i, N} \in[0,1 / N]$ for all $i=0, \ldots, N-1$. Consequently

$$
\frac{1}{N} \sum_{i=0}^{N-1} \nu_{i, N}^{2} \leq \frac{1}{N} \sum_{i=0}^{N-1} \frac{1}{N^{2}}=\frac{1}{N^{2}} \underset{N \rightarrow \infty}{\stackrel{a . s .}{\longrightarrow}} 0 .
$$

Finally, by (5.1), (5.2) and (5.3) the result is achieved.

Acrayaknowledgements: This research was partially supported by Project MATHAMSUD 19-MATH-06 and 18-MATH-07. H.Araya was partially supported by Proyecto FONDECYT Post-Doctorado 3190465, N. Bahamonde was partially supported by FONDECYT Regular 1201898, T. Roa was partially supported by Beca CONICYT-PFCHA/Doctorado Nacional/201821180298, S. Torres was partially supported by FONDECYT 1171335.

\section{References}

Araya H., Bahamonde N., Fermin L., Roa T. and Torres S.(2021). On the consistency of the least squares estimator in models sampled at random times driven by long memory noise: the renewal case Statistica Sinica, DOI number: 10.5705/ss.202020.045\%..

Araya H., Bahamonde N., Fermin L., Roa T. and Torres S.(2019). Parameter estimation for random sampled Regression Model with Long Memory Noise arXiv:1902.08590. 


\section{REFERENCES}

Baillie, R.T. and Chung, S.K. (2002). Modeling and forecasting from trend-stationary long memory models with applications to climatology. International Journal of forecasting, 18:205226.

Bardet, J.M. and Pierre R. Bertrand (2010) . A non-parametric estimator of the spectral density of a continuous-time Gaussian process observed at random times. Scandinavian Journal of Statistics, 37(3):458-476.

Bellhouse, D.R. (1981). Area estimation by point-counting techniques. Biometrics, pages 303312.

Brockwell, A.E. (2007). Likelihood-based Analysis of a Class of Generalized Long-Memory Time Series Models. International Journal of Business 86 Economic Statistics, 28(3):386-407.

Chang, C. (2014). Optimum preventive maintenance policies for systems subject to random working times, replacement, and minimal repair. Computers $\&$ Industrial Engineering, $67: 185-194$

Khan, M., (2017). Performance testing of computed radiography system and imaging plates. In ASNT Annual Conference 2017, 83-92.

Krune, E., Krueger, B., Zimmermann, L., Voigt, K., and Petermann, K. . (2016). Comparison of the jitter performance of different photonic sampling techniques. Journal of Lightwave Technology, 34(4):1360-1367.

Lobato, I. and Velasco, C. (2000). Long Memory in Stock-Market Trading Volume. International Journal of Business \& Economic Statistics, 18(4):410-427. 


\section{REFERENCES}

Mandelbrot, B. and Van Ness, J. (1968). Fractional brownian motions, fractional noises and applications. SIAM review, 10(4):422-437.

Masry, E. (1983). Probability density estimation from sampled data. IEEE Trans. Inform. Theory, 29(5):696-709.

Max-Moerbeck, W., Richards, J. L., Hovatta, T., Pavlidou, V., Pearson, T. J., and Readhead, A. C. S. . (2014). A method for the estimation of the significance of cross-correlations in unevenly sampled red-noise time series. Monthly Notices of the Royal Astronomical Society, 445(1):437-459.

Nieto-Barajas, L. and Sinha, T. (2015). Bayesian interpolation of unequally spaced time series. Stochastic environmental research and risk assessment, 29(2):577-587.

Ólafsdóttir, KB., Schulz, M., and Mudelsee, M. (2016). Redfit-x: Cross-spectral analysis of unevenly spaced paleoclimate time series. Computers \& Geosciences, 91:11-18.

Philippe, A., Robet, C. \& Viano, M. (2020). Random discretization of stationary continuous time processes. Metrika https://doi.org/10.1007/s00184-020-00783-1.

Roa, T., Torres, S. and Tudor, C. (2020). Limit distribution of the least square estimator with observations sampled at random times driven by standard Brownian motion. In arXiv:2012.08966, Submitted, Preprint.

Subr, K., Nowrouzezahrai, D., Jarosz, W., Kautz, J., and Mitchell, K. (2014). Error analysis of estimators that use combinations of stochastic sampling strategies for direct illumination.

In Computer Graphics Forum, volume 33, 93-102. Wiley Online Library. 


\section{REFERENCES}

Tudor C.A. (2008). Analysis of the Rosenblatt process. In ESAIM: Probability and statistics, volume $12,230-257$.

Tudor C.A. (2013). Analysis of variations for self-similar processes: A stochastic calculus approach. Springer Science \& Business Media.

Vilar, J.A. (1995). Kernel estimation of the regression function with random sampling times. Test, 4(1):137-178.

Vilar, J.A., and Vilar, J.M. (2000). Finite sample performance of density estimators from unequally spaced data. Statistics and Probability Letters, 50:63-73.

Zhao, X., Chen, M. and Nakagawa, T. (2014). Optimal time and random inspection policies for computer systems. Appl. Math, 8(1L):413-417. 